Original Research Paper

\title{
Optimal Pareto Solutions of a Dynamic $C$ Chart: An Application of Statistical Process Control on a Semiconductor Devices Manufacturing Process
}

\author{
${ }^{1}$ Toni Lupo and ${ }^{2}$ Filippo Sgroi \\ ${ }^{I}$ Dipartimento di Ingegneria Chimica, Gestionale, Informatica, Meccanica (DICGIM), \\ ${ }^{2}$ Department of Agricultural and Forest Sciences, \\ Università degli Studi di Palermo, 90128 Palermo, Italy
}

Article history

Received: 23-02-2015

Revised: 31-03-2015

Accepted: 22-05-2015

Corresponding Author:

Toni Lupo

Dipartimento di Ingegneria

Chimica, Gestionale,

Informatica, Meccanica

(DICGIM), Università degli

Studi di Palermo, 90128

Palermo, Italy

Email: toni.lupo@unipa.it

\begin{abstract}
The present paper proposes a novel economic-statistical design procedure of a dynamic $c$ control chart for the Statistical Process Control (SPC) of the manufacturing process of semiconductor devices. Particularly, a non-linear constrained mathematical programming model is formulated and solved by means of the $\varepsilon$-constraint method. A numerical application is developed in order to describe the Pareto frontier, that is the set of optimal $c$ charts and the related practical considerations are given. The obtained results highlight how the performance of the developed dynamic $c$ chart overcome that of the related static one, thus demonstrating the effectiveness of the proposed procedure.
\end{abstract}

Keywords: Statistical Process Control, Dynamic $C$ Chart, Multi-Objective Design, $\varepsilon$-Constraint Method, Semiconductor Devices Manufacturing

\section{Introduction}

The quality control of a manufacturing process represents a crucial and critical issue in every modern manufacturing context. Statistical Process Control (SPC) and Process Capability Analysis (PCA) applications and procedures are widely considered to perform quality control of a manufacturing process (Lupo, 2015a). These strategies appear to be important in the globalized business environment (Di Trapani et al., 2014; Sgroi et al., 2014a; 2014b; Lupo, 2015b).

Referring to SPC operations, control charts represent the main tool to analyze the inherent variability of a process output and to achieve and maintain the in-control process state (Woodall and Montgomery, 2014). Many charts schemes are available with reference to peculiarities of the under control process output (Montgomery, 2013). For example, considering the manufacturing process of semiconductor devices, the SPC operations mainly concern the evaluation of notfunctioning devices in a production run (Qin et al., 2006; Lee et al., 2006) and, in such a condition, the control chart for non-conformities numbers in inspection units, also called $c$ chart, is typically considered.

In the present paper, a novel multi-objective design procedure for an enhanced scheme of the $c$ chart is developed. In particular, a dynamic $c$ chart is considered, i.e., a chart scheme whose parameters can be changed during SPC operations to adapt chart characteristics to different process situations, in order to improve the chart effectiveness (Zimmer et al., 2000; Inghilleri et al., 2015). In detail, the developed design procedure takes into account two objectives to be optimized:

- The statistical one, to indirectly optimize the immeasurable or even hard to evaluate quality cost part, which is strongly related to the statistical performance of the employed chart (Lupo, 2014)

- The economic one, to also take care of the measurable quality cost portion

More in detail, the considered goal is to determine the parameters of a dynamic $c$ chart in order to minimize both the hourly total quality costs (economic objective) and the out-of-control average run length of the chart (statistical objective), i.e., the expected number of points required by the chart to indicate an out-of-control state of the process. A non-linear constrained mathematical model is formulated to solve the treated multi-objective problem whereas the optimal chart configurations, that is the Pareto frontier, are obtained by means of the $\varepsilon$-constraint method. Finally, the effectiveness of the proposed procedure is shown in a numerical application as regards to SPC operations related to a manufacturing process of semiconductor devices. 
The reminder of the paper is organized as follows: The theoretical aspects of the treated problem are presented first, followed with the description on the model to evaluate the economic performance of control charts; the multi-objective mathematical model is formulated next. Meanwhile, the application of the proposed procedure is presented in detail. Hence, conclusion of this study is provided as well.

\section{Fundamental Definitions}

A dynamic $c$ chart to monitor the non-conformities number $x$ of a manufacturing process output is considered. In detail, in such a type of chart the zone within the control limits $[-\mathrm{k}, \mathrm{k}]$ is divided into three strips. Two zones are defined: The central zone, $[-\mathrm{w}, \mathrm{w}]$ and the warning zones, $[-\mathrm{k},-\mathrm{w}) \mathrm{U}(\mathrm{w}, \mathrm{k}]($ Fig. 1$)$. If the point representative of the $t^{\text {th }}$ sample statistic $c$ is plotted within the central zone, then the next one will have a sample size of $n_{1}$ and will be taken $h_{1}$ time unit after the $t^{\text {th }}$ one. On the contrary, if the point representative of the $t^{\text {th }}$ sample $c$ is plotted within the warning zone, then the next one will have a sample size of $n_{2}>n_{1}$ and will be taken $h_{2}<h_{1}$ time unit after the $t^{\text {th }}$ one. Finally, if the point representative of the $t^{\text {th }}$ sample is plotted outside of the control limits, then the out-of-control signal has to be considered as a false alarm or as a consequence of the occurrence of an out-of-control cause.

As well known, the under control non-conformities number $x$ follows the Poisson probability distribution with parameter $\bar{c}>0$, which represents its mean and variance. However, when such parameter exceeds 10 , the normal distribution $N(\bar{c}, \bar{c})$ can be considered as a satisfactory approximation of the Poisson distribution with parameter $\bar{c}$. Therefore, in such a condition, the $c$ chart can be plotted considering the standard normal distribution $Z \sim N(0,1)$ and, in particular, the following sample statistic can be considered:

$$
Z_{i}=\frac{x_{i}-\bar{c}}{\sqrt{(\bar{c} / n)}}
$$

In which, $x_{i}$ is the observed average non-conformities number in the sample $i$ composed by $n$ inspection units. Finally, the process in-control time is assumed to be exponentially distributed with mean $1 / \lambda$ and the of outof-control condition occurrence determines an instantaneous and persistent shift magnitude $\delta$ in the process mean $\bar{c}$.

\section{Cost Model}

The total quality costs $C_{\mathrm{T}}$ arising from the employment of a control chart can be expressed as:

$C_{T}=C_{L R}+C_{Q}(\delta)$

where, $C_{\mathrm{LR}}$ and $C_{\mathrm{Q}}(\delta)$ are the hourly labor resource and quality control costs over a system functioning cycle, respectively. In the next section, the system functioning cycle is defined and all the durations of its periods are evaluated, whereas computations of $C_{\mathrm{LR}}$ and $C_{\mathrm{Q}}$ costs are formulated next.

\section{System Functioning Cycle}

The process functioning cycle is supposed to be constituted by the following fundamental periods (Duncan, 1956):

- $\quad$ The in-control period $P_{\text {in }}$

- $\quad$ The out-of-control period $P_{\text {out }}$

- The time $T_{1}$ required to detect the assignable cause and the time $T_{2}$ to restore the system

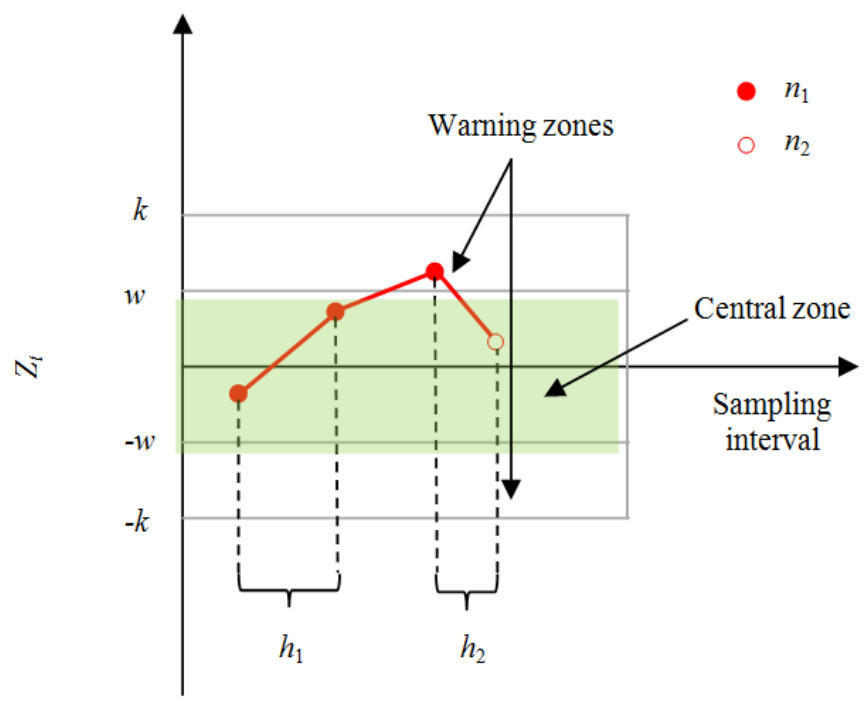

Fig. 1. Dynamic c chart with two zones 
The expected length of a system functioning cycle $T(\delta)$ represents the period between two successive starting of the manufacturing process, after detecting the out-of-control assignable cause and solving its related problems.

The in-control period length $P_{\text {in }}$ is given by:

$P_{i n}=\frac{1}{\lambda}+\frac{\left(1-d_{1}\right) \cdot s \cdot T_{0}}{A R L_{0}}$

where, $\lambda$ is the failure rate of the manufacturing system, while the second term takes into account the false alarms effects over the in-control period. Let $d_{1}$ be a binary variable that takes 1 if the production continues during assignable cause searches and 0 otherwise, $T_{0}$ the expected search time associated to a false alarm of the chart, $A R L_{0}$ the in control average run length, i.e., the expected number of points plotted on the chart before a point indicates a false out-ofcontrol condition and $s$ the expected number of samples taken during the in-control period.

To the contrary, the out-of-control period has a time duration $P_{\text {out }}$ given by the following equation:

$P_{\text {out }}=\left(E_{\delta}(h)-\zeta\right)+E_{\delta}(h) \cdot\left(A R L_{\delta}-1\right)+\frac{E_{\delta}(n)}{r_{I N}}$

where, $E_{\delta}(h)$ is the out-of-control expected value of sampling interval, $A R L_{\delta}$ is the out-of-control average run length of the chart and the last term represents the time to inspect the sample providing the out-of-control signal, i.e., the last sample in the system functioning cycle. $E_{\delta}(n)$ is the expected value of the sample size while the process is out-of-control and $r_{I N}$ the sample inspection rate. Thus, according to Equations 3 and 4, the expected length of the system functioning cycle $T(\delta)$ assumes the following expression:

$T(\delta)=\frac{1}{\lambda}+\frac{\left(1-d_{1}\right) \cdot s \cdot T_{0}}{A R L_{0}}+A T S_{\delta}+\frac{E_{\delta}(n)}{r_{I N}}+T_{1}+T_{2}$

The functions of $s, A R L_{0}, A R L_{\delta}, A T S_{\delta}, E_{\delta}(n), E_{0}(h)$ and $E_{\delta}(h)$ are given with more details in Lupo (2014).

\section{Labor Resources Cost $C_{\mathrm{LR}}$}

For the adaptive aforementioned policy, the needed labor resources capacity can vary at two levels, $L R_{1}$ and $L R_{2}$, according to the chart parameters stated at each sampling epoch. In particular, let $i$ be the zone of the control chart which the point representative of the $(t-1)^{\text {th }}$ sample has been plotted in under the assumption that the entire sampling interval $h_{i}$ is used to inspect the sample of size $n_{i}$. The amount of labor resources needed to inspect the next $t^{\text {th }}$ sample can be obtained by:

$$
L R_{i}=\frac{n_{i}}{h_{i} \cdot r_{I N}} \text { for } i=1,2
$$

Therefore, the expected amount of Labor Resources $E(L R)$ can be evaluated as the weighted sum of $L R_{1}$ and $L R_{2}$ by the related in-control probabilities that a point is plotted on the chart within the zone 1 or 2 (Lupo, 2015c). Consequently, the expected labor resources cost $C_{\mathrm{LR}}$ can be computed by the following Equation 7:

$C_{\mathrm{LR}}=E(L R) \cdot c_{\mathrm{LR}}$

Being $c_{\mathrm{LR}}$ the hourly labor resources cost.

\section{Quality Control Costs $C_{\mathrm{Q}}(\delta)$}

The hourly quality control cost related to the out-ofcontrol shift $\delta, C_{\mathrm{Q}}(\delta)$, can be obtained by dividing costs arising from the quality control in a system functioning cycle $C_{\delta}$ by the system cycle time $T(\delta)$, that is:

$C_{Q}(\delta)=\frac{C_{\delta}}{T(\delta)}$

The costs $C_{\delta}$ can be computed by means of the following Equation 9:

$$
C_{\delta}=C_{1}+C_{2}(\delta)+C_{3}+C_{4}(\delta)
$$

Where:

$C_{1}$ and $C_{2}(\delta)=$ The costs arising from the nonconforming products manufactured during the system in-control and out-ofcontrol periods, respectively

$C_{3} \quad=$ The cost due to the false alarms, the detection and the removing of out-of control causes

$C_{4}(\delta)=$ The cost arising from the sampling activities required by the control chart

The costs $C_{1}$ and $C_{2}(\delta)$ represent the economic loss due to the deviation of the production output from its target value $T$. As suggested by Wu et al. (2004), such an economic loss can be effectively estimated by the quadratic representation of the quality loss function via the following relationships:

$$
\begin{aligned}
& C_{1}=P_{\text {in }} \cdot r_{P R} \cdot \int_{T}^{+\infty} c_{n c}(x-T)^{2} f(x) d x \\
& C_{2}(\delta)=P_{\text {out }} \cdot r_{P R} \cdot \int_{T}^{+\infty} c_{n c}(x-T)^{2} f_{\delta}(x) d x
\end{aligned}
$$

In which $f(x)$ and $f_{\delta}(x)$ are the probability density functions of normal distributions $N(\bar{c}, \bar{c})$ and $\mathrm{N}\left(\mathrm{c}_{\delta}, \mathrm{c}_{\delta}\right)$ 
when the process is in-control and out-of-control respectively. The term $r_{P R}$ is the production rate (units/h) and $c_{n c}$ is a constant depending on the external cost associated with the production of non-conforming parts.

The cost $C_{3}$ in Equation 9 is given by the following Equation 12:

$$
C_{3}=\frac{s \cdot Y}{A R L_{0}}+W
$$

In which $Y$ and $W$ are the costs associated with the false alarms of the chart and the detection and the removal of out-of-control causes, respectively.

Lastly, the sampling cost $C_{4}(\delta)$ is given by:

$$
C_{4}(\delta)=\left(a+b \cdot E_{0}(n)\right) \cdot s+\left(a+b \cdot E_{\delta}(n)\right) \cdot \mathrm{s}^{\prime}
$$

where, $a$ and $b$ are the sampling cost components, $E_{0}(n)$ is the expected value of the sample size while the process is in-control and $s$ and $s$ ' are the expected number of samples taken during the system in-control and out-of-control periods, respectively. The formulation of $s^{\prime}$ and $E_{0}(n)$ are detailed in Lupo (2014).

\section{Multi-Objective Problem Formulation}

Aiming at optimizing the dynamic $c$ chart parameters, the following mixed integer non-linear constrained mathematical model is formulated:

$$
\begin{aligned}
& \min C_{T}\left(n_{1}, n_{2}, h_{1}, h_{2}, w, k\right) \\
& \min A R L_{\delta}\left(n_{1}, n_{2}, h_{1}, h_{2}, w, k\right)
\end{aligned}
$$

Subjected to the following constraints:

$A R L_{0} \geq L$

$L R_{\min } \leq L R_{1} \leq L R_{2} \leq L R_{\max }$

$n_{\min } \leq n_{1}<n_{2} \leq n_{\max }$

$h_{\min } \leq h_{2}<h_{1} \leq h_{\max }$

$k_{\min } \leq w<k \leq k_{\max }$

In detail:

- (16) ensures a minimum required in-control average run length of the chart

- (17) implies that a minimum part of the available labor resource should be employed to perform the SPC and, at the same time, that the labor resource does not exceed the available resource capacity
- (18)-(20) assure the adaptive capability of the dynamic $c$ chart and limit the sampling effort

The considered objective functions (14) and (15) contrast one each other so that it is not possible to find out a single optimal solution corresponding to the best result for both the considered objectives. Thus, in Multi-Objective Optimization Problems (MOOPs), as that one considered here, the concept of solutions dominance has to be applied. More in detail, a generic solution $X_{1}$ of a MOOP dominates the generic solution $X_{2}$ if $X_{1}$ is non-worse than $X_{2}$ for each objective and $X_{1}$ is strictly better than $X_{2}$ for at least one objective (Erfani and Utyuzhnikov, 2011).

In the literature, various resolution methods have been developed to point out optimal solutions of a MOOP (Certa et al., 2014). The simplest and probably the most widely used approach to solve a MOOP is the $\varepsilon$-constraint method (Marler and Arora, 2004). The latter consists in reformulating the considered MOOP by taking into account only one of its objectives and restricting the remaining ones within user-chosen values of the parameter $\varepsilon$. By applying this resolution method, the MOOP is thus reduced to a single-objective optimization problem and the set of non-dominated solutions can be obtained in more than one optimization steps. Particularly, in order to describe the Pareto frontier for the MOOP here considered the Lexicographic Goal Programming (LGP) method (Deb, 2001) is initially used to find out the extreme Pareto frontier solutions. For example, these sequential steps allow to find out the extreme solution (A) of the minimum total quality costs (Fig. 2):

- Minimizing $C_{\mathrm{T}}$ function (Equation 14) as a single objective optimization problem obtaining $C_{\mathrm{T}}$,min

- Minimizing $A R L_{\delta}$ function (Equation 15), by imposing that $C_{\mathrm{T}}$ has to take a value not greater than $C_{\mathrm{T}, \min }$, obtaining $A R L_{\delta, \max }$

This procedure is analogously applied changing the objectives hierarchy to find out the other two bounds $\left(A R L_{\delta, \min }, C_{\mathrm{T}, \max }\right)$ of the extreme solution (B).

Once the extreme points of the Pareto frontier are determined, the $\varepsilon$-constraint method has to be recursively applied. In the first step, the $C_{\mathrm{T}}$ function is minimized and the $A R L_{\delta}$ is forced to take a value smaller than the $A R L_{\delta \text {,max }}$ previously obtained. In this way, the optimal solution (1) $\left(A R L_{\delta, 1} ; C_{\mathrm{T}, 1}\right)$ belonging to the Pareto frontier can be found. In the next step, such a procedure is repeated minimizing the $C_{\mathrm{T}}$ function and imposing that the $A R L_{\delta}$ function has to take a value smaller to that one corresponding to the solution previously found. The procedure is repeated until the other Pareto frontier extreme (B) is obtained. In such way, the $\varepsilon$-constraint method ensures the determination of the whole Pareto frontier (Certa et al., 2011; Lupo, 2015d). 


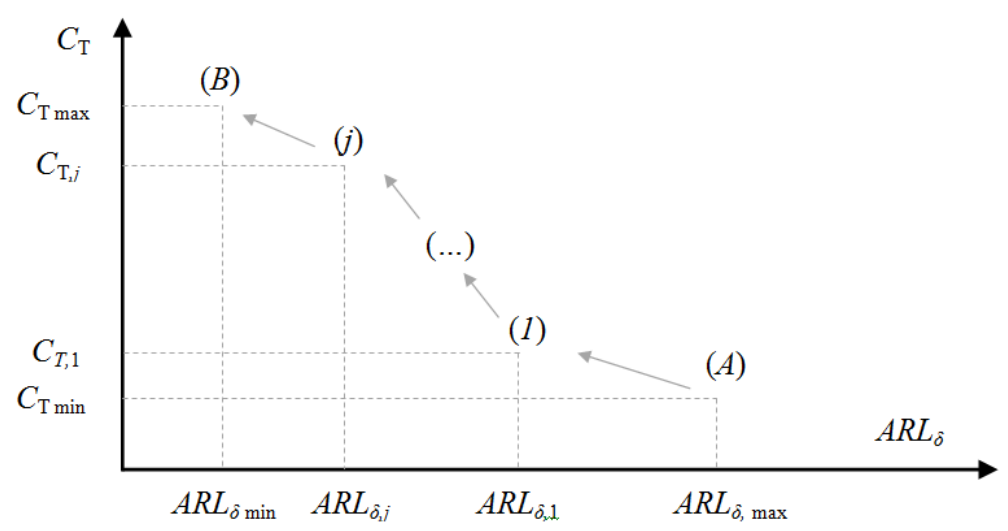

Fig. 2. $\varepsilon$-constraint multi-step optimization procedure

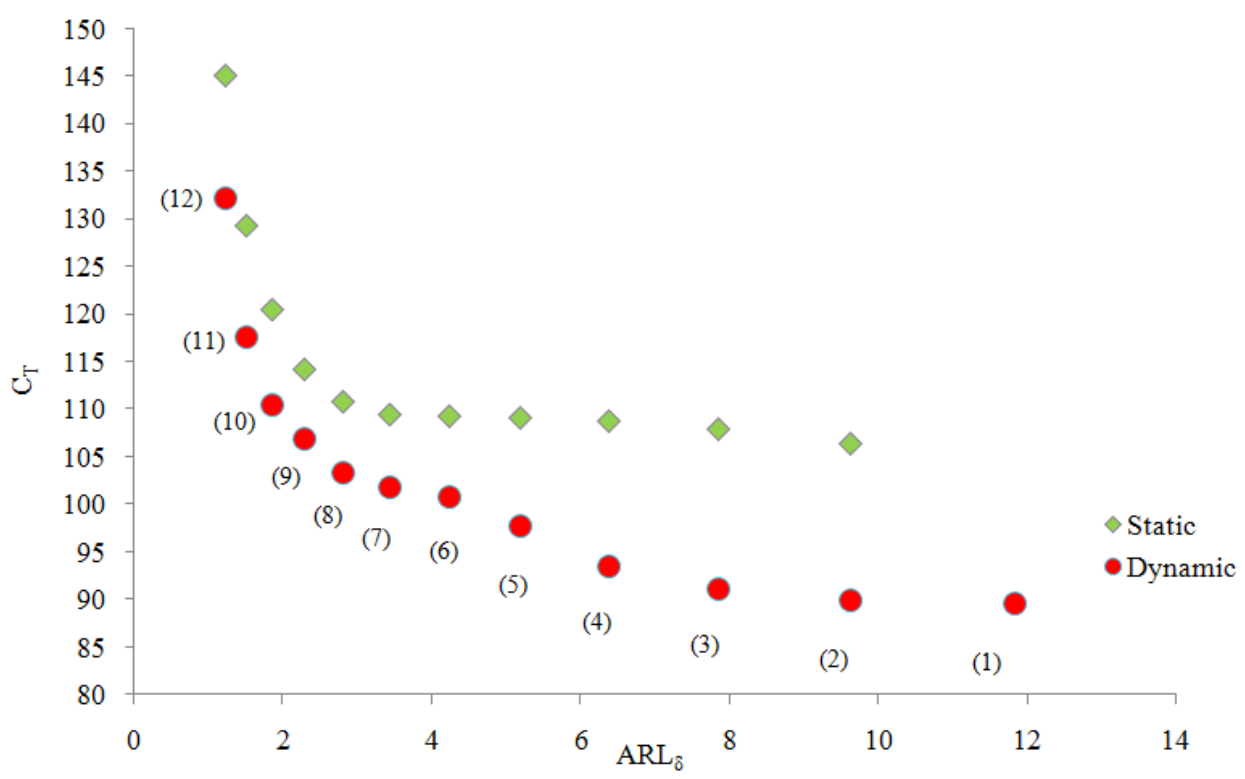

Fig. 3. Optimal Pareto frontiers of dynamic and static c chart schemes $\left(A R L_{0}=600 ; \delta=1\right)$

\section{Application: Control Quality of Manufacturing Process of Semiconductor Devices}

The manufacturing process of semiconductor devices, such as diodes, transistors, integrated circuits, microprocessors and different kind memories (RAM and ROM), follows a multi-step sequence of photo lithographic and chemical processing steps during which electronic circuits are gradually created on a wafer made of pure semiconducting material. Normally, the fundamental process parameters are monitored during manufacturing operations to promptly diagnose incipient process faults or malfunctions and SPC operations are performed on the process output by monitoring the nonconformities number in the whole production outcome or in its samples. Such an activity, which is considered a significant overall measure of manufacturing process effectiveness, is commonly performed by means of the classical static $c$ control chart (Cubberly and Bakerjian, 1989; Bhatt, 2000; May and Spanos, 2006).

In this section, the multi-objective design procedure previously developed is adopted to point out the optimal configurations of the dynamic $c$ chart. In particular, a firm working in the memories manufacturing field for Solid State Devices (SSD) in herein considered. Table 1 reports the related operating and costs parameters.

The non-linear Generalized Reduced Gradient Algorithm (GRG) implemented by the Microsoft Excel ${ }^{\circledR}$ solver has been used to solve the multi-objective optimization problem. By minimizing both objective functions, namely the total quality costs $C_{\mathrm{T}}$ (Equation 14) and the expected out-of-control average run length $A R L_{\delta}$ (Equation 15), the chart parameters have been obtained.

Table 2 the Pareto frontier compared with the one related to the typical static scheme of the $c$ chart is shown in Fig. 3. 
Table 1. Numerical application data

\begin{tabular}{lllr}
\hline$a(\$)$ & 0 & $T_{0}(\mathrm{~h})$ & 0.083 \\
$b(\$ /$ measure $)$ & 0.001 & $T_{l}(\mathrm{~h})$ & 0.083 \\
$c_{n c}(\$ /$ non-conforming part $)$ & 20 & $T_{2}(\mathrm{~h})$ & 0.750 \\
$c_{L R}(\$ / \mathrm{h})$ & 10 & $d_{1}$ & 1.000 \\
$r_{P R}($ units/h) & 100 & $d_{2}$ & 0.000 \\
$r_{I N}($ measures $/ \mathrm{h})$ & 5 & $Y(\$)$ & 2.000 \\
$\lambda($ failure/h) & 0.05 & $W(\$)$ & 700.000 \\
$A R L_{0}$ & 600 & & 1.000 \\
$\bar{c}$ & 10 & & $\delta$ \\
Bounds of the design variable & & & \\
$n_{\min } ; n_{\max }$ & $1 ; 50$ & \\
$h_{\min } ; h_{\max }(\mathrm{h})$ & $0.1 ; 50$ & & \\
$w_{\min } ; w_{\max }$ & $1 ; 5$ & \\
$K_{\min }$ & $w+0.2$ & \\
$L R_{\min } ; L R_{\max }($ Resource number $)$ & $0.01 ; 3$ & & \\
\hline
\end{tabular}

Table 2.Optimal dynamic $c$ chart configurations $\left(A R L_{0}=\right.$ $600 ; \delta=1)$

\begin{tabular}{lllllll}
\hline \multicolumn{7}{c}{$600 ; \delta=1)$} \\
Optimal & \multicolumn{7}{l}{ Chart parameter value } & & & \\
solution & $n_{1}$ & \multicolumn{1}{c}{$n_{2}$} & $h_{1}$ & $h_{2}$ & $w$ & $k$ \\
\hline 1 & 1 & 8 & 0.32 & 0.32 & 1.79 & 3.14 \\
2 & 1 & 9 & 0.36 & 0.36 & 1.70 & 3.14 \\
3 & 1 & 11 & 0.44 & 0.44 & 1.69 & 3.14 \\
4 & 1 & 12 & 0.48 & 0.48 & 1.56 & 3.14 \\
5 & 1 & 14 & 0.56 & 0.56 & 1.46 & 3.14 \\
6 & 6 & 6 & 1.24 & 0.36 & 1.86 & 3.14 \\
7 & 7 & 7 & 1.48 & 0.41 & 1.92 & 3.14 \\
8 & 8 & 8 & 1.73 & 0.46 & 1.93 & 3.14 \\
9 & 10 & 10 & 2.12 & 0.58 & 2.09 & 3.14 \\
10 & 11 & 11 & 2.62 & 0.50 & 1.84 & 3.14 \\
11 & 14 & 14 & 3.21 & 0.57 & 1.95 & 3.14 \\
12 & 20 & 20 & 4.31 & 0.80 & 2.09 & 3.14 \\
\hline
\end{tabular}

From Fig. 3, the Pareto frontier regarding the herein proposed dynamic $c$ chart dominates that one corresponding to the related typical static configuration. In detail, from the economic point of view, the optimal dynamic $c$ chart scheme is represented by the solution (1) with $A R L_{\delta}=11.83$ and $C_{\mathrm{T}}=89.58 \$$. To the contrary, from the statistical performance point of view, the optimal solution (12), with $A R L_{\delta}=1.23$ and a $C_{\mathrm{T}}=132.2$ $\$$, represents the related best one. In addition, Table 2 shows that with the increasing of the statistical performance required to the optimal chart configurations, from solution (1) on, also the sample sizes ( $n_{1}$ and $\left.n_{2}\right)$ and the sampling intervals $\left(h_{1}\right.$ and $\left.h_{2}\right)$ increase with no severe changes in the chart control limits $w$ and $k$. Finally, if the Pareto optimal frontier includes lots of solutions, the decision maker could be supported by a decision multi-criteria method as the Analytic Hierarchy Process (AHP) (Saaty, 1990) or the ELimination Et ChoixTRaduisant la REalitè (ELECTRE) (Roy, 1978) in order to select the chart configuration that represents the best compromise among the considered objectives.

\section{Conclusion}

A multi-objective design procedure for a dynamic $c$ chart has been developed with the aim to point out the suitable optimal chart configurations to perform SPC operations on a manufacturing process of semiconductor devices. The problem has been formulated by a non-linear constrained mathematical model and the $\varepsilon$-constraint method has been employed to describe the Pareto frontier. The knowledge of this frontier will provide useful information to the decision maker about the decisional process that cannot be directly obtained by the typically considered design procedures. For instance, if a budget constraint is added, the sub-set of the optimal feasible solutions on which restricting the selection can be immediately individuated. Moreover, the frontier analysis could address the decision maker to discard some solutions which imply small cost reductions with meaningful statistical performance decreases (such as solutions (1), (2) and (3)) or, to the contrary, small statistical improvements with significant cost increments (such as solutions (11) and (12)). Finally, the developed approach is simple to be used, it is fully supported by an Excel macro and it requires short computational time.

\section{Acknowledgement}

The authors would thanks to the considered firm working providing the numerical data employed to develop the treated application.

\section{Funding Information}

The authors did not receive any private or public funding to develop the research.

\section{Author's Contributions}

This study is a result of the full collaboration of all the authors. 
Toni Lupo: Wrote paragraphs Fundamental Definitions, Multi-Objective Problem Formulation and Conclusion.

Filippo Sgroi: Wrote paragraphs Introduction and Application: Control Quality of Manufacturing Process of Semiconductor Devices.

\section{Ethics}

The authors have no conflicts of interest in the development and publication of current research.

\section{References}

Bhatt, G.D., 2000. Organizing knowledge in the knowledge development cycle. J. Knowl. Manage., 4: 15-26. DOI: 10.1108/13673270010315371

Certa, A., G. Galante, T. Lupo and G. Passannanti, 2011. Determination of Pareto frontier in multi-objective maintenance optimization. Reliab. Eng. Syst. Safety, 96: 861-867. DOI: 10.1016/j.ress.2010.12.019

Certa, A., Lupo, T., Passannanti, G., 2014. An efficient proposal for the application of simulated annealing algorithms. 1st International Conference on Engineering and Applied Sciences Optimization, OPTi 2014; Kos Island; Pages 910-927; Code 108723.

Cubberly, W.H. and R. Bakerjian, 1989. Tool and Manufacturing Engineers Handbook Desk Edition. 1st Edn., Society of Manufacturing Engineers, Dearborn, ISBN-10: 0872633519, pp: 1250.

Deb, K., 2001. Multi-Objective Optimization Using Evolutionary Algorithms. 1st Edn., John Wiley \& Sons, Chichester, ISBN-10: 047187339X, pp: 497.

Di Trapani, A.M., R. Squatrito, M. Foderà, R. Testa and S. Tudisca et al., 2014. Payment for environmental services for the sustainable development of the territory. Am. J. Environ. Sci., 10: 480-488. DOI: 10.3844 /ajessp.2014.480.488

Duncan, A.J., 1956. The economic design of X charts used to maintain current control of a process. J. Am. Statistical Associat., 51: 228-242. DOI: $10.1080 / 01621459.1956 .10501322$

Erfani, T. and S.V. Utyuzhnikov, 2011. Directed search domain: A method for even generation of the Pareto frontier in multiobjective optimization. Eng. Optimizat., 43: 467-484.

DOI: $10.1080 / 0305215 X .2010 .497185$

Inghilleri, R., T. Lupo and G. Passannanti, 2015. An effective double sampling scheme for the control chart. Quality Reliab. Eng. Int., 31: 205-216. DOI: $10.1002 /$ qre. 1572

Lee, D.E., I. Hwang, C.M. Valente, J.F.G. Oliveira and D.A. Dornfeld, 2006. Precision manufacturing process monitoring with acoustic emission. Int. J. Machine Tools Manufacture, 46: 176-188.

DOI: 10.1016/j.ijmachtools.2005.04.001
Lupo, T., 2014. Economic-statistical design approach for a VSSI X-BAR chart considering Taguchi loss function and random process shifts. Int. J. Reliab. Quality Safety Eng., 21: 1450006. DOI: $10.1142 / \mathrm{S} 0218539314500065$

Lupo, T., 2015a. The new Nino capability index for dynamic process capability analysis. Quality Reliab. Eng. Int., 31: 305-312. DOI: 10.1002/qre.1589

Lupo, T., 2015b. Fuzzy ServPerf model combined with ELECTRE III to comparatively evaluate service quality of international airports in Sicily. J. Air Transport Manage., 42: 249-259. DOI: 10.1016/j.jairtraman.2014.11.006

Lupo, T., 2015c. Economic robustness analysis of adaptive chart schemes for monitoring the total nonconformities number in inspection units. Int. J. Reliab. Quality Safety Eng., 22: 1550002 DOI: $10.1142 / \mathrm{S} 0218539315500023$

Lupo, T., 2015d. Non-dominated "trade-off" solutions in television scheduling optimization. Int. Trans. Operat. Res., 22: 563-584.

DOI: 10.1111 itor. 12137

Marler, R.T. and J.S. Arora, 2004. Survey of multiobjective optimization methods for engineering. Structural Multidisciplinary Optimizat., 26: 369-395. DOI: $10.1007 / \mathrm{s} 00158-003-0368-6$

May, G.S. and C.J. Spanos, 2006. Fundamentals of Semiconductor Manufacturing and Process Control. 1st Edn., John Wiley \& Sons, Hoboken, ISBN-10: 0471790273, pp: 428.

Montgomery, D.C., 2013. Introduction to Statistical Quality Control. 1st Edn., John Wiley \& Sons, ISBN-10: 1118610024.

Qin, S.J., G. Cherry, R. Good, J. Wang and C.A. Harrison, 2006. Semiconductor manufacturing process control and monitoring: A fab-wide framework. J. Process Control, 16: 179-191. DOI: 10.1016/j.jprocont.2005.06.002

Roy, B., 1978. ELECTRE III: Un algorithme de classementsfondesurune representation floue des preference en presence de criteres multiples. Cahiers de CERO, 20: 3-24.

Saaty, T.L., 1990. How to make a decision: The analytic hierarchy process. Eur. J. Operat. Res., 48: 9-26. DOI: 10.1016/0377-2217(90)90057-I

Sgroi, F., A.M. Di Trapani, R. Testa and S. Tudisca, 2014a. Strategy to increase the farm competitiveness. Am. J. Agric. Biol. Sci., 9: 394-400. DOI: 10.3844 /ajabssp.2014.394.400

Sgroi, F., S. Tudisca, A.M. Di Trapani, R. Testa and R. Squatrito, 2014b. Efficacy and efficiency of Italian energy policy: The case of PV systems in greenhouse farms. Energies, 7: 3985-4001. DOI: $10.3390 /$ en 7063985 
Woodall, W.H. and D.C. Montgomery, 2014. Some current directions in the theory and application of statistical process monitoring. J. Quality Technol., 46: 78-94.

Wu, Z., M. Shamsuzzaman and E.S. Pan, 2004. Optimization design of control charts based on Taguchi's loss function and random process shifts. Int. J. Product. Res., 42: 379-390.

DOI: $10.1081 / 00207540310001614169$
Zimmer, L.S., D.C. Montgomery and G.C. Runger, 2000. Guidelines for the application of adaptive control charting schemes. Int. J. Product. Res., 38: 1977-1992. DOI: $10.1080 / 002075400188447$ 